\title{
A Fuzzy Reasoning System for Boundary Detection in Radiologycal Images
}

\author{
J.M. Molina, M.J. Martín, P. Isasi, A. Sanchis \\ Dpto. Informática, Univ.Carlos III de Madrid, MADRID,SPAIN \\ Tel: (34)(1)6249455, Fax: (34)(1)6249430,E-mail: molina@ia.uc3m.es
}

\begin{abstract}
In this paper, a new method to edge detection in blurred images, like radiological ones, has been developed. It is based on fuzzy systems, in order to incorporate the expert knowledge about the images. Fuzzy logic is used to obtain the edge of a bone in a radiological image without being forced to adjust the system. Classical algorithms, like Sobel filter, obtain similar results, but the final user needs to modify different parameters in order to adapt the accuracy of the resulting image.

The robustness of the fuzzy system allows in a second step to process the resulting image in order to detect different shapes. This second process will be used for automatic medical analysis. An illustrative computational fuzzy edge detector system has been implemented and its performance evaluated.
\end{abstract}

\section{Introduction}

Many medical problems need the exhaustive examination of radiological images to evaluate some diseases [1]. In those problems the human expert ought to analyse some features of a blurred image, the radiological one, in order to extract the needed information. The development of an automatic system that allow the evaluation of radiological images involves four major steps: image acquisition, token extraction, feature computation and analysis.

From the image acquired in the first step, the characteristics, tokens, of the images are extracted in the second step. The result of the token extraction is a simplified image that contains all main features [2]. This new image is useful in third step in order to find relations between the extracted features. The final step takes a decision about diseases analysing the relations.

Classical methods of image processing, used in token extraction, need to previously adjust some parameters when are applied to blurred images [3]. Therefore, the simplified image obtained in second step needs human cooperation to adjust the parameters. After that, following steps can be done automatically. A real automatic system should extract the token information from different radiological images without human intervention. In this case the system ought to resemblance the logical procedure of the human expert in order to obtain the main information from the image.

A fuzzy-symbolic [4] new methodology to cope with the token extraction problem that appears in radiological images is proposed in this paper. This fuzzy system has been applied in a particular real problem that system is described in Section 2. To reproduce the judgement of a human observer, which is based on subjective, qualitative descriptions of the perceived features, a rule-based fuzzy system will be used. In Section 3 , the formalism to obtain a symbolic measure of tokens existence, in particular edge tokens, is described. The edge point possibility in a radiological image defines a possible bone which shape must be examined. The proposed approach has been implemented on a fuzzy system that is described in Section 4. Some results are shown in Section 5. Finally some conclusions are presented.

\section{Problem Description}

The evaluation of radiological images requires some subjetive information about the objects that appears in the image. Radiological images of dogs have been studied in this work. The analysis of bones in an animal is determinated to define the dog race and the animal genealogy in order to prevent and treat many diseases.

In this case the automatic system ought to be able to recognize the curvature of the bone. The description of this curvature allows to define the pureness grade, pedigree, of the dog race. When bone head of a femur, see Figure 1, has a perfectly circular shape the dog is recognize as pure race. 


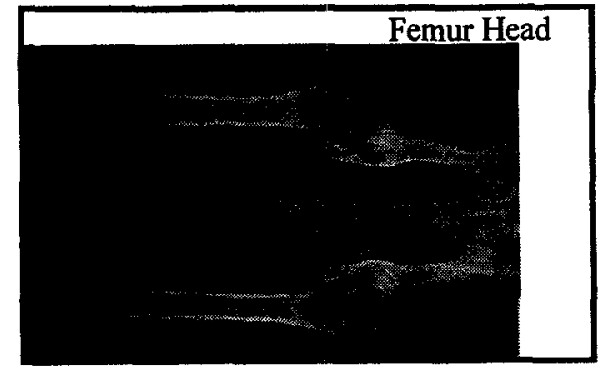

Figure 1. Radiological image of a dog femur.

To make automatic decisions about the race, the edge extraction filter ought to obtain a non-blured image with the edge perfectly defined. This image will allow to apply an automatic algorithm that claculates the curvature of femur head.

Classical image extraction edge algorithms, like the Sobel one, in a blurred image give many possible points as edges. In this case the head bone is over defined, and many points could belong to the head bone and the computational effort is huge to extract the circular shape of the bone. Modifying the threshold of the edge extractor it is possible to obtain less number of points. However an specific adjustment of the filter has to be done to each image. This task requires the collaboration of a human expert.

The aim of this work is to avoid the human interaction by means of a fuzzy system, able to extract the minimum number of points keeping the edge perfectly defined.

\section{Fuzzy Edge Evaluation}

To automate the pursued human-like edge extraction process, the first step should be to select adequate descriptions of intensity values, "analogous" to those formulated by humans when interpreting radiological images. Artificial Intelligence representation schemes best suit these requirements. A generic pixel will be represented as a set of intensity values of the neigbours pixels, $\left(I_{1}, I_{2}, \ldots, I_{n}\right)$, set of values characterizing the central pixel; each $f_{i} \in F_{i}$, being $F_{i}$ the range of possible numerical values of $f_{j}$.

Edge characteristic of the pixel can then be related with a metric distance in the space of possible values. Given a numerical distance, $d_{i}$, between $f_{i}$ (values from the central and neigbours pixels, respectively), $D_{i}$ is defined as the range of all possible values of $d_{j}$. To better cope with the intrinsic uncertainty of the perceived features, numerical values of the distances $d_{i}$ will be mapped into qualitative symbolic descriptions. Thus, the computed distances will be transformed, through a fuzzification process, into linguistic variables [4]. For each $d_{i}, a$ linguistic variable, $L d_{i}$, is introduced together with its set of values $\left\{\mathrm{ld}_{\mathrm{i} 1}, \mathrm{ld}_{\mathrm{i} 2}, \ldots, \mathrm{ld}_{\mathrm{ic}}\right\}$, each one labelling a fuzzy subset in $\mathrm{D}_{\mathrm{i}}$ (its meaning). The fuzzification operation applied to the numerical distances $d_{i}$, will result in their transformation into a fuzzy singleton [4], fuzzy subset whose membership function is the Kronecker delta, $\delta\left(d_{-}-d_{i}\right)$, in $D_{i}$.

A fuzzy relational algorithm (FRA) [4] will store the knowledge required to obtain the possibility of an edge characteristic of the pixel, based on the linguistic similarities between the central pixel and its neigbours. The FRA will be composed by a finite set of fuzzy conditional statements of the form IF $\operatorname{AND}^{n}{ }_{i=1}\left(\operatorname{Ld}_{i}\right.$ is $\operatorname{ld}_{i j_{i}}$ ) THEN (LEDGE is $\mathbf{l s}_{\mathbf{k}}$ ), whose antecedents are conjunctions of fuzzy statements about the linguistic variables $\operatorname{Ld}_{i}(i=1,2, \ldots, n)$, and their consequents fuzzy statements about LEDGE, linguistic edge central pixel, whose set of values is $\left\{\mathrm{ss}_{1}, \mathrm{ls}_{2}, \ldots, \mathrm{s}_{\mathrm{p}}\right\}$. The well known Mamdani implication [5] has been chosen to assign their meaning to these fuzzy conditional statements.

The compositional rule of inference (CRI) [4], (approximate extension of the rule of modus ponens) has been adopted as the inference mechanism to obtain the fuzzy subset induced in LEDGE (through each conditional statement of the FRA), by the fuzzy statenent of the form $A N D^{n_{i}=1}\left(L_{i} d_{i}\right.$ is $\left.1 d_{i r}\right)$, linguistic description of the distance among the intensity of surrounding pixels. The meaning of LEDGE will be the intersection of the intermediate meanings resulting from the application of the CRI to each conditional statement of the FRA ( $\mathrm{min}$ of all the induced consequent membership functions). Finally, the adopted defuzzification process, applied to the final meaning of LEDGE, will be the traditional Centre of Gravity procedure [6].

\section{Fuzzy System Developed}

The fuzzy system developed considers each image pixel and their eight-neighbours as it is shown in Figure 2.

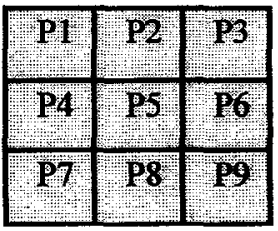

Figure 2. $3 \times 3$ Array centred on P5.

The system inputs are the intensity distance among the pixels defined in this way:

Horizontal $=|\mathrm{P} 6-\mathrm{P} 4|+|\mathrm{P} 5-\mathrm{P} 4|$

Vertical $=|\mathbf{P 8}-\mathbf{P} 2|+|\mathbf{P} 5-\mathbf{P} 2|$ 
Right-Left Diagonal $=\mid$ P3 - P7 $|+|$ P5 - P7 $\mid$

Left-Right Diagonal $=|\mathrm{P} 1-\mathrm{P} 9|+|\mathrm{P} 5-\mathrm{P} 9|$

Centre $=$ P5

The system output is the type of the central pixel. Then the system output is a linguistic variable named: Output Pixel.

Figure 3 shows the memberships function of inputs. The values range of the system inputs goes from 0 to 265, and on this range the labels: Very Small, Small, Large, Very Large, are defined.

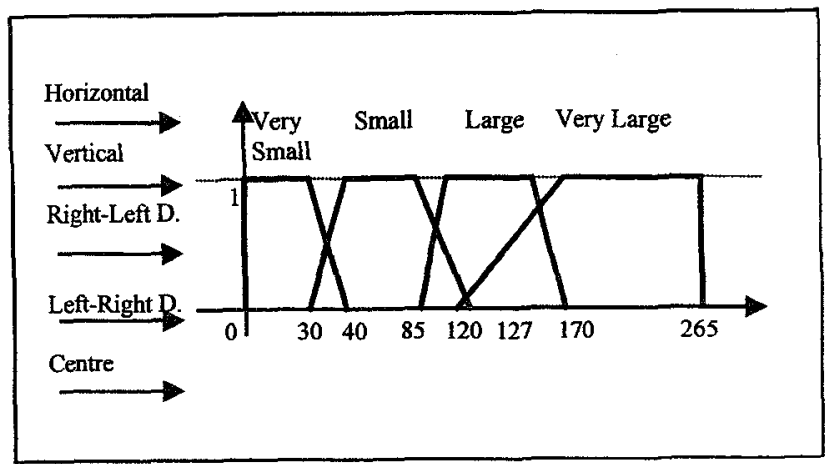

Figure 3. Memberships function of inputs.

The next step is find a rule system able to get all boundaries in the image, the figure 4 shows these rules.



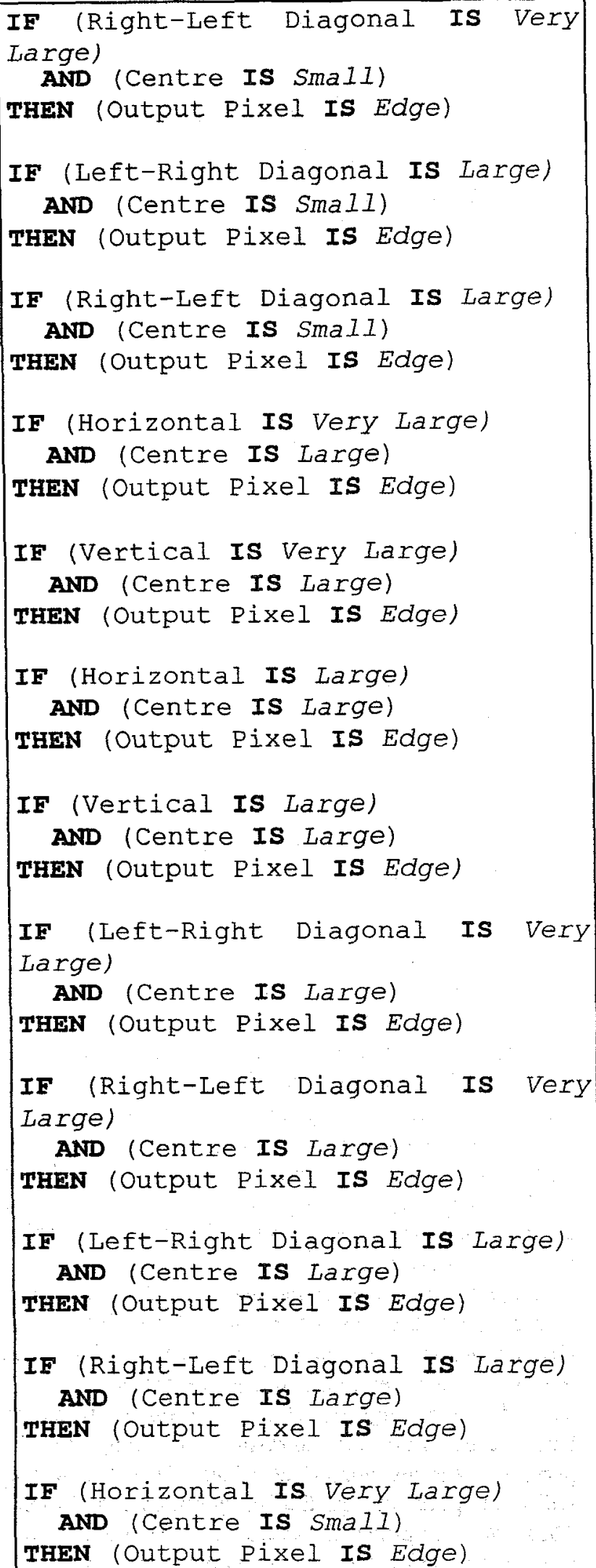




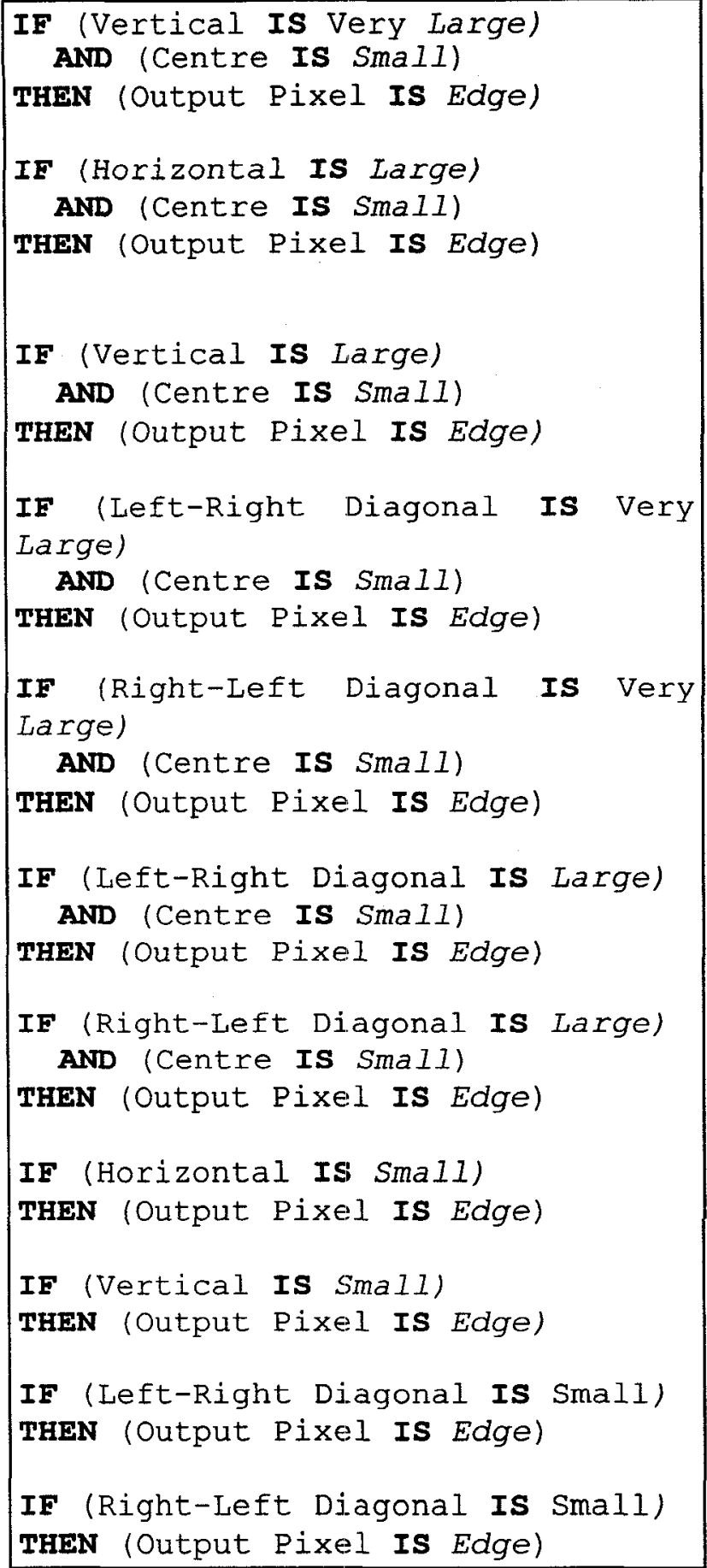

Figure 4. Rules used in the Fuzzy System

The figure 5 shows the memberships functions outputs. It can be seen that there is only a label: Edge.

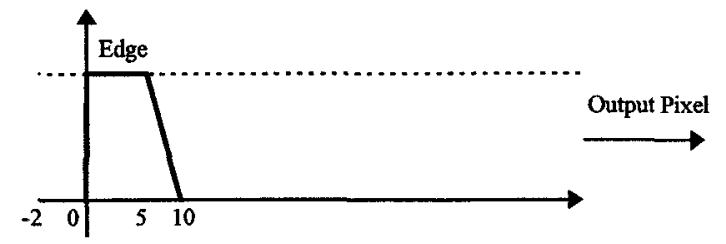

Figure 5. Memberships function of outputs.

\section{Experiments and Results}

The above described system has been tested with images from dog bone radiographs. The processing of these images is not simple because a background of varying grey level exits.

The results will be compared with the outputs of Quick Sobel Operator that depends on a threshold. The level of the threshold defines the sensibility in order to obtain a certain amount of edge pixels.

In Figures 6, 7 y 8 some results are shown, each one has a different type of original image and shows the resulting image for the fuzzy system and two Sobel operators:

(a) shows the original image

(b) shows the boundary found by fuzzy technique

(c) shows the boundary found by Quick Sobel Operator with a threshold of 10

(d) shows the boundary found by Quick Sobel Operator with a threshold of 20 .

In Figure 6, it can be seen the first example of the experiments, in this case the image is a type 1 radiography.

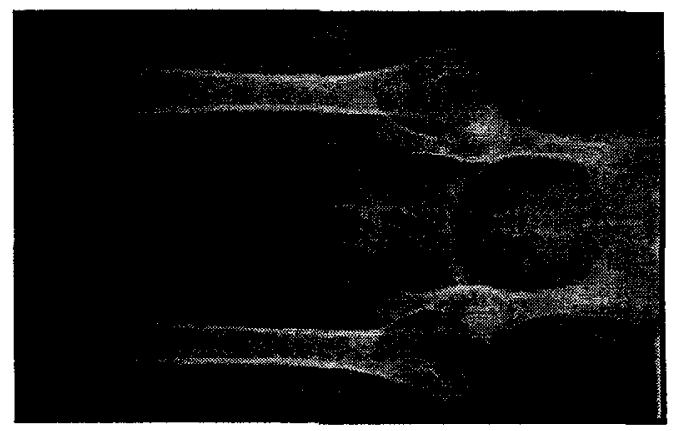

(a) Original image 


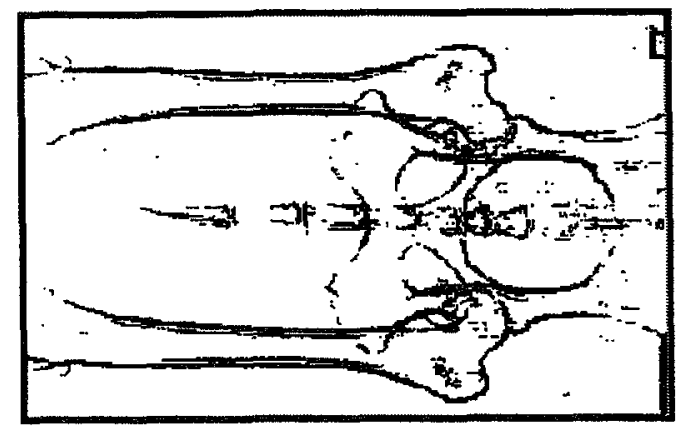

(b) Fuzzy system

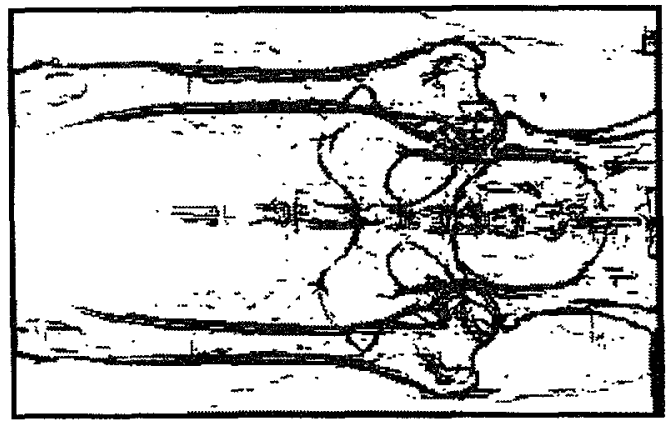

(c) Sobel 10

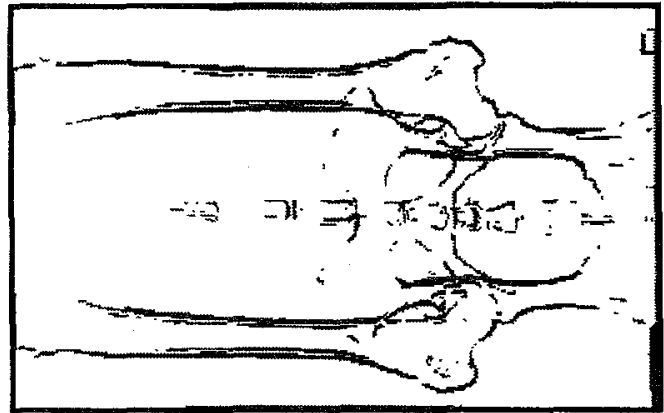

(d) Sobel 20

Figure 6. Radiological image type 1.

Figure 6 (c) shows the image boundaries well defined but there is some noise present and "spurious" boundaries. Unfortunately, if either of these conditions is present, it will be difficult extract the desired features from the image. Figure 6 (d) shows the image boundaries badly defined, therefore this method does not give the required information. Figure 6 (b) shows the most appropriate technique to find edges around regions of differing grey level in this type of radiological images. Then we can see another type of images (Figure 7 and Figure 8) where the fuzzy method is more efficient than the other ones. Many different images of the three types has been analysed, and the results are similar to the ones show in this figures.

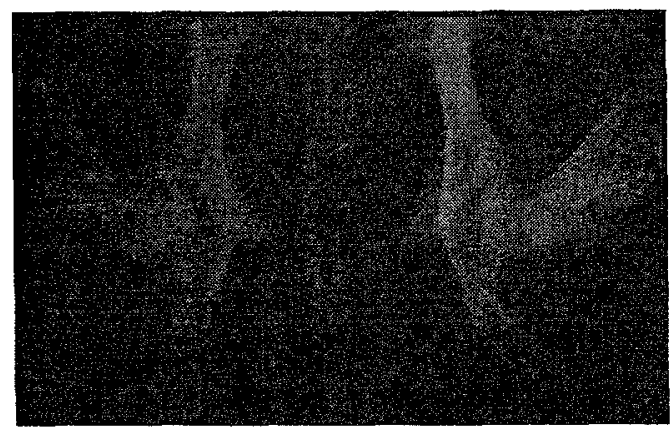

(a) Original image

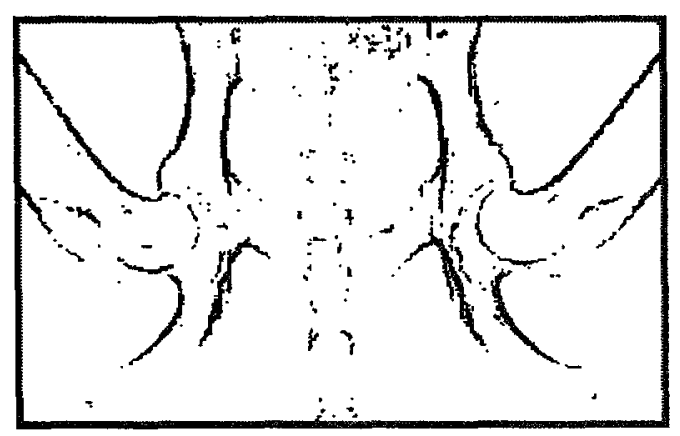

(b) Fuzzy system

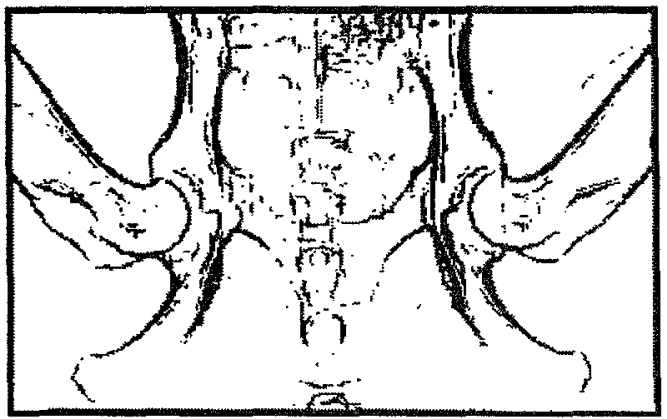

(c) Sobel 10

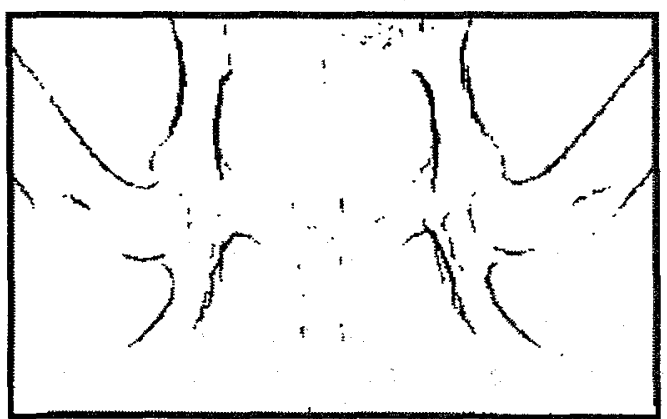

(d) Sobel 20

Figure 7. Radiological image, type 2. 


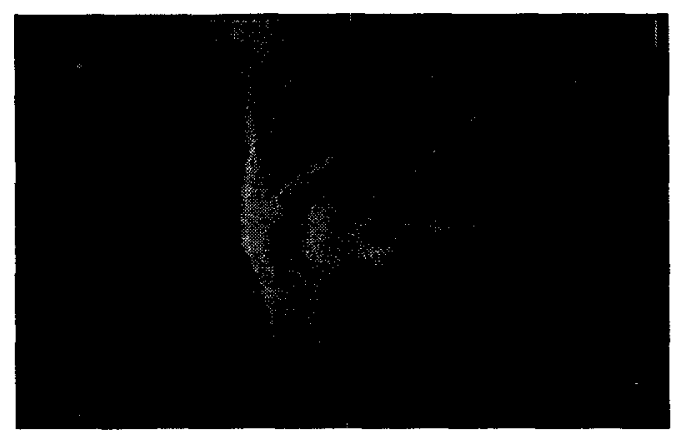

(a) Original image

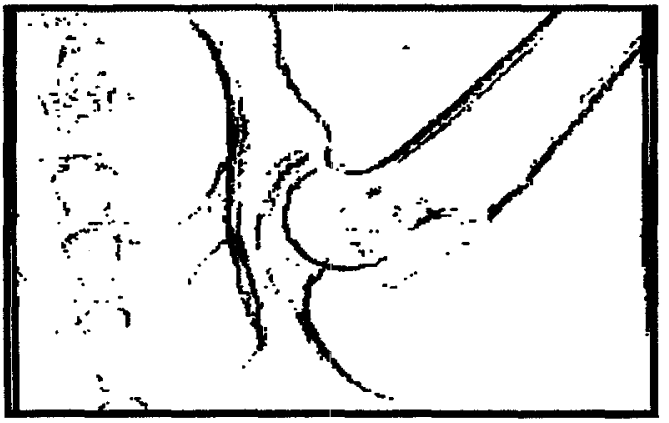

(b) Fuzzy system

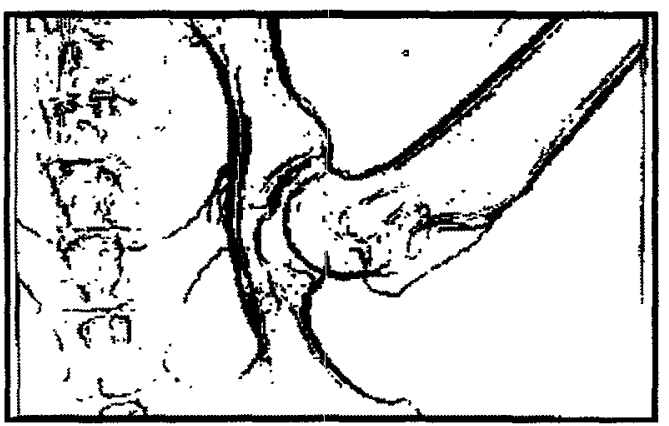

(c) Sobel 10

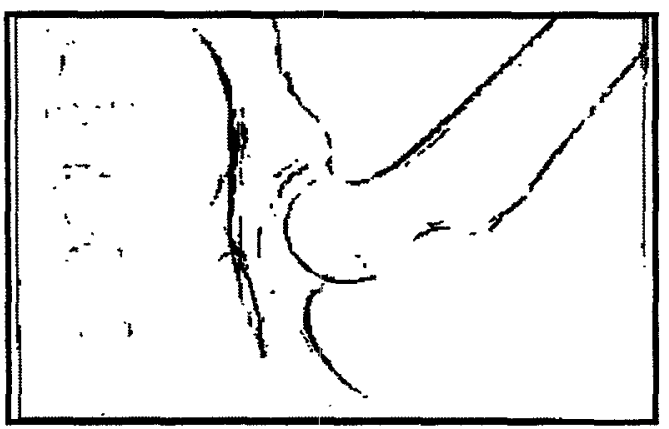

(d) Sobel 20

Figure 8. Radiological image type 3.
Although the results could be very similar between the fuzzy system and the quick operator Sobel, this last traditional method requires the adjustment of the appropriate threshold level depending on the image being processed. The search of the threshold value can be a slowly and unsatisfactory process.

\section{Conclusions}

A new methodology, based on fuzzy logic concepts, has been introduced to model the edge extraction. Through the use of the type of linguistic descriptions employed by humans, we improve the capability to deal with the edge detection problem, which may be considered too complex to be analysed with conventional algorithms. The proposed methodology, completely applicable to other types of images, could be the basis of new hierarchical multilevel applications suitable for implementation on a blackboard architecture [7].

\section{References}

[1] Ashkar, G.P. and Modestiono J.W., "The contour extraction problem with biomedical applications", CGIP 7, 1978, 331-335.

[2] A. K. Jain, Fundamentals of Digital Image Processing, Ed. Prentice-Hall, 1989.

[3] Ballard D.H. and Brown C.M., Computer Vision, Ed. Prentice-Hall, 1982.

[4] L. A. Zadeh, "Outline of a New Approach to the Analysis of Complex Systems and Decision Processes", Trans. on SMC, Jan. 1973.

[5] Mamdani H., "Aplication of Fuzzy Logic to Aproximate Reasoning Using Linguistic Synthesis", IEEE Trans. on Computers, Nov/Dic. 1988.

[6] Zimmermann H.J., Fuzzy Sets, Uncertainty and Information, Kluwer 1985.

[7] G. F. Luger, W. A. Stubblefield, Artificial Intelligence. Structures and Strategies for Complex Problem Solving, The Benjamin/Cummings Publ., 1993. 